Eur. J. Clin. Chem. Clin. Biochem.

Vol. 30, 1992, pp. 325-331

(C) 1992 Walter de Gruyter \& Co. Berlin · New York

\title{
Biosynthesis of Guanidinoacetic Acid in Isolated Renal Tubules
}

\author{
By Michio Takeda ${ }^{1}$, Ikuo Kiyatake ${ }^{1}$, Hikaru Koide ${ }^{1}, K_{y u}$ Yong Jung ${ }^{2}$ and Hitoshi Endou ${ }^{2}$ \\ ${ }^{1}$ Division of Nephrology, Department of Medicine, Juntendo University School of Medicine \\ 2 Department of Pharmacology, Faculty of Medicine, University of Tokyo, Tokyo, Japan
}

(Received October 30, 1991/February 24, 1992)

Summary: Guanidinoacetic acid, a precursor of creatine, is an essential substrate for muscle energy metabolism. Since guanidinoacetic acid has been reported to be synthesized from arginine and glycine by glycine amidinotransferase (transamidinase) in kidney homogenates or slices, the purpose of this study was to provide evidence of guanidinoacetic acid synthesis in isolated tubules from rat kidneys, and to clarify the mechanism regulating it. Isolated rat tubules were incubated with various substrates. Guanidinoacetic acid was separated by high performance liquid chromatography and measured fluorometrically.

Results obtained were as follows:

(1) Guanidinoacetic acid was synthesized from arginine or canavanine and glycine in isolated rat tubules.

(2) $D, L$-Norvaline, ornithine and methionine suppressed guanidinoacetic acid synthesis.

(3) Creatine suppressed guanidinoacetic acid synthesis, i.e. creatine was a negative feedback inhibitor of guanidinoacetic acid synthesis in this in vitro system.

(4) Guanidinoacetic acid was not synthesized from hydroxyurea, citrulline, argininosuccinic acid or canaline.

These data demonstrate that guanidinoacetic acid is synthesized only from arginine or canavanine and glycine, and that the guanidine cycle may not function fully in the rat renal tubule.

\section{Introduction}

Guanidinoacetic acid is synthesized by glycine amidinotransferase ${ }^{1}$ ) (transamidinase) mainly in the kidney (1). Part of the guanidinoacetic acid synthesized in the kidney is transported to the liver, where it is methylated to creatine (2) and the rest is excreted in the urine (3).

Guanidinoacetic acid itself has been reported to have certain physiological roles, e.g., the stimulation of

\footnotetext{
1) Glycine-amidinotransferase (EC 2.1.4.1)

Ornithine transcarbamylase (EC 2.1.3.3)

Argininosuccinic acid synthetase (EC 6.3.4.5)

Argininosuccinic acid lyase (EC 4.3.2.1)

Guanidinoacetate methyltransferase (EC 2.1.1.2)

Carbamylphosphate synthetase (EC 6.3.4.16)

Arginase (EC 3.5.3.1)
}

insulin secretion (4). However, the biological significance of guanidinoacetic acid in the body is most apparent when guanidinoacetic acid synthesis is decreased in the kidney. In chronic renal failure, the decrease of guanidinoacetic acid synthesis in the kidney results in a decreased creatine content in various organs, including skeletal muscle (5). On the other hand, guanidinoacetic acid excretion in the urine is closely correlated with creatinine clearance (6). Urinary excretion of guanidinoacetic acid, which may reflect a metabolic function of the kidney, is decreased when renal function deteriorates (3), and the measurement of urinary guanidinoacetic acid excretion has been reported to be an effective index for the detection of renal dysfunctions such as gentamicin nephropathy (7), hypertensive renal disease (8) and rejection of renal transplantation (9). 
Arginine or canavanine plus glycine serve as substrates for guanidinoacetic acid synthesis in rat kidney homogenates $(10-15)$ and slices (1). Transamidinase ${ }^{1}$ ) activity in the kidney is regulated by hormones $(16)$ or dietary factors $(16,17)$ in vivo. Creatine feeding was reported to suppress transamidinase activity in the kidney $(16,17)$.

Ungar et al. (18) and Fitch et al. (19) added growth hormone and creatine, respectively, to a guanidinoacetic acid synthesizing system in rat kidney homogenates, but could not reproduce the in vivo phenomenon. Furthermore, only one study has demonstrated the regulatory mechanism of guanidinoacetic acid synthesis in vitro, in which ornithine suppressed guanidinoacetic acid synthesis in rat kidney homogenates (10).

The purpose of this study was to investigate the mechanism of guanidinoacetic acid synthesis in vitro by using various substrates in isolated renal tubules from rat kidneys. We propose a possible pathway for the regulation of guanidinoacetic acid synthesis.

\section{Materials and Methods}

\section{Animals}

Male Sprague-Dawley rats (Charles River, Tokyo, Japan) weighing 200 to $250 \mathrm{~g}$ were used in these experiments.

\section{Biochemical materials}

Collagenase (type I), bovine serum albumin (fraction V), canavanine, canaline and argininosuccinic acid were purchased from Sigma Chemical (St. Louis, MO). Arginine, glycine, $D, L$ norvaline, ornithine and creatine were purchased from Wako Pure Chemical (Osaka, Japan). Hydroxyurea and citrulline were purchased from Tokyo Kasei Kogyo (Tokyo, Japan). All other chemicals were of analytical grade and purchased from commercial sources.

\section{Isolation procedure for renal tubules}

Rat renal tubules were isolated by the method of Obara et al. (20). In brief, rats were anaesthetized by the intraperitoneal administration of $50 \mathrm{mg} / \mathrm{kg}$ body weight phenobarbital. The left kindney was perfused in situ through the renal artery with ice-cold Hanks' solution $\left(1 \mathrm{mmol} / 1 \mathrm{CaCl}_{2}\right.$ ) containing $1 \mathrm{~g} / \mathrm{l}$ collagenase (Sigma type I) and $1 \mathrm{~g} / \mathrm{l}$ bovine serum albumin. Kidney slices, $1 \mathrm{~mm}$ in thickness, were prepared and incubated at $37^{\circ} \mathrm{C}$ for $30 \mathrm{~min}$ in the same solution bubbled with $95 \%$ oxygen gas. After incubation, the slices were washed with ice-cold Hanks' solution $\left(0.25 \mathrm{mmol} / \mathrm{l} \mathrm{CaCl}_{2}\right)$. The mixture was then stirred with a vortex mixer for $15 \mathrm{~s}$, and the upper turbid medium was transferred to a $50 \mathrm{ml}$ centrifuge tube (Corning Laboratory Science Co., NY). This mechanical dispersion procedure was repeated three times. The particles of dissociated tissue were removed by filtration through a $1 \mathrm{~mm}$ nylon mesh. After allowing the suspension containing tubules and cells to stand for 5 min in ice, the upper fraction containing almost all cells was carefully pipetted out. The precipitate was resuspended in Hanks' solution and centrifuged at $300 \mathrm{~g}$ for $5 \mathrm{~min}$. The pellet was resuspended in Hanks' solution and used as a tubular suspension.
Assay of guanidinoacetic acid synthesized in isolated renal tubules

The isolated tubules were incubated in $1 \mathrm{ml} \mathrm{Hanks}$ ' solution containing various substrates with shaking at $60 \mathrm{~min}^{-1}$ in a 1.5 $\mathrm{ml}$ microtube (Sarstedt, Germany) at $37^{\circ} \mathrm{C}$. At the end of each incubation, the tissue was disintegrated by pulsatile sonication for $30 \mathrm{~s}$ with a Sonifier (Branson Sonic Power Co., Conn. USA), followed by the addition of $89 \mu \mathrm{l}$ of $1 \mathrm{~kg} / \mathrm{l}$ trichloroacetic acid. The trichloroacetic acid extracts were filtered through a 0.45 $\mu \mathrm{m}$ Millipore filter (Millipore Limited Japan). The $\mathrm{pH}$ of the aliquots was adjusted to 2.2 with $2 \mathrm{~mol} / \mathrm{l} \mathrm{NaOH}$. Aliquots of the adjusted extracts were used for guanidinoacetic acid determination.

Guanidinoacetic acid was separated by high performance liquid chromatography (HPLC) using a Guanidinopack II (Japan Spectroscopic Co., Tokyo, Japan), eluted with $0.2 \mathrm{~mol} / 1$ sodium citrate buffer, $\mathrm{pH} \mathrm{3.0,} \mathrm{at} \mathrm{a} \mathrm{flow} \mathrm{rate} \mathrm{of} 0.5 \mathrm{ml} / \mathrm{min}$ for $10 \mathrm{~min}$, followed by $0.2 \mathrm{~mol} / \mathrm{l}$ sodium citrate buffer, $\mathrm{pH} 3.5$, at $0.5 \mathrm{ml} /$ $\min$ for $5 \mathrm{~min}$, and determined fluorometrically using 9,10phenanthrenequinone for the post-labelling method (21). Fluoroscence was measured (excitation at $365 \mathrm{~nm}$, emission at 495 nm) with a fluorescence detector (Model FP 110-C, Japan Spectroscopic Co., Tokyo, Japan). The areas of chromatographic peaks were determined with a Chromatocorder 12 (System Instrument Co., Tokyo, Japan) with a baseline display connected between the fluoromonitor and the recorder. The tissue protein concentration was determined by the method of Lowry et al. (22).

\section{Calculation and statistics}

The ratio of the area of each sample in HPLC to the standard area was calculated. Amounts of guanidinoacetic acid are shown as $\mu \mathrm{mol} / \mathrm{g}$ tissue protein. All results are expressed as mean \pm SE. Statistical analyses were performed by the MannWhitney rank sum test (23).

\section{Results}

Guanidinoacetic acid synthesis in isolated renal tubules

We incubated the isolated renal tubules with various concentrations of arginine or canavanine and $1 \mathrm{mmol} / 1$ glycine. As shown in figure 1 , the doseresponse relationship between guanidinoacetic acid synthesis and arginine was observed in the range of 0 to $2.0 \mathrm{mmol} / \mathrm{l}$ of arginine. Guanidinoacetic acid synthesis from canavanine was saturated by less than $2 \mathrm{mmol} / \mathrm{l}$ of canavanine (data not shown). The isolated renal tubules were also incubated with various concentrations of glycine and $1 \mathrm{mmol} / \mathrm{l}$ arginine. The dose-response relationship between glycine and guanidinoacetic acid synthesis was observed in the range from 0 to $2.0 \mathrm{mmol} / \mathrm{l}$ of glycine (data not shown). Furthermore, we incubated the isolated renal tubules with $1 \mathrm{mmol} / 1$ arginine or $1 \mathrm{mmol} / \mathrm{l}$ canavanine and $1 \mathrm{mmol} / \mathrm{l}$ glycine for $0-90 \mathrm{~min}$. As shown in figure 2 , guanidinoacetic acid synthesis increased up to 90 min, depending on the incubation time. 


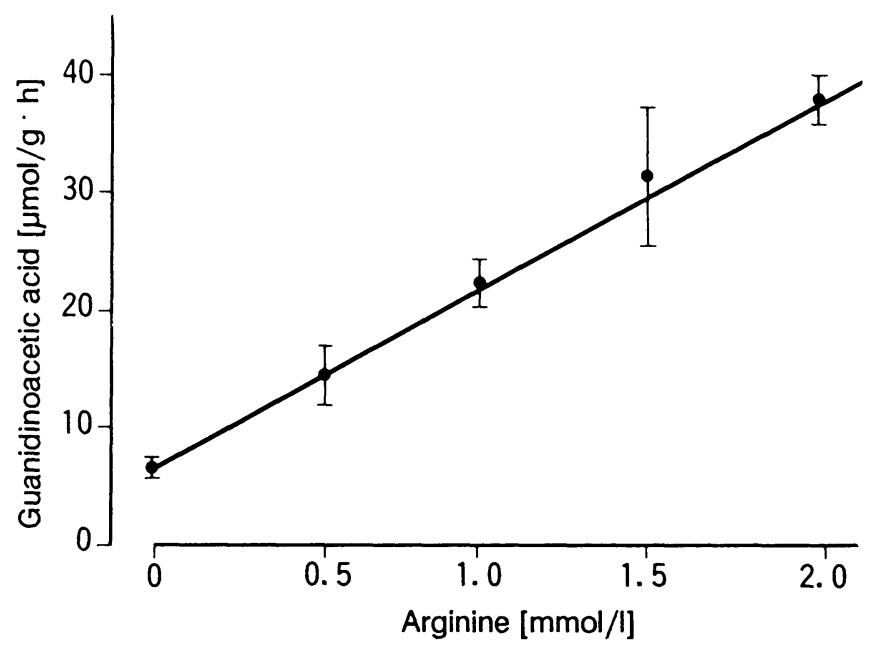

Regulation of guanidinoacetic acid synthesis by various substrates in the isolated renal tubules

$D, L$-Norvaline and ornithine have been reported to suppress transamidinase activity in human pancreas homogenates (24) and isolated rat hepatocytes (25). Methionine also suppresses guanidinoacetic acid synthesis in isolated rat hepatocytes (25). However, there has been only one report concerning the regulation of the guanidinoacetic acid synthesis in the kidney; Ratner et al. (10) reported that ornithine suppressed

Fig. 1. Dose-response curve of arginine concentration versus guanidinoacetic acid synthesis in isolated renal tubules. The isolated tubules were incubated for $60 \mathrm{~min}$ with various concentrations of arginine and $1 \mathrm{mmol} / \mathrm{l}$ glycine. Values are means \pm SE of 4 samples $(r=0.999)$.
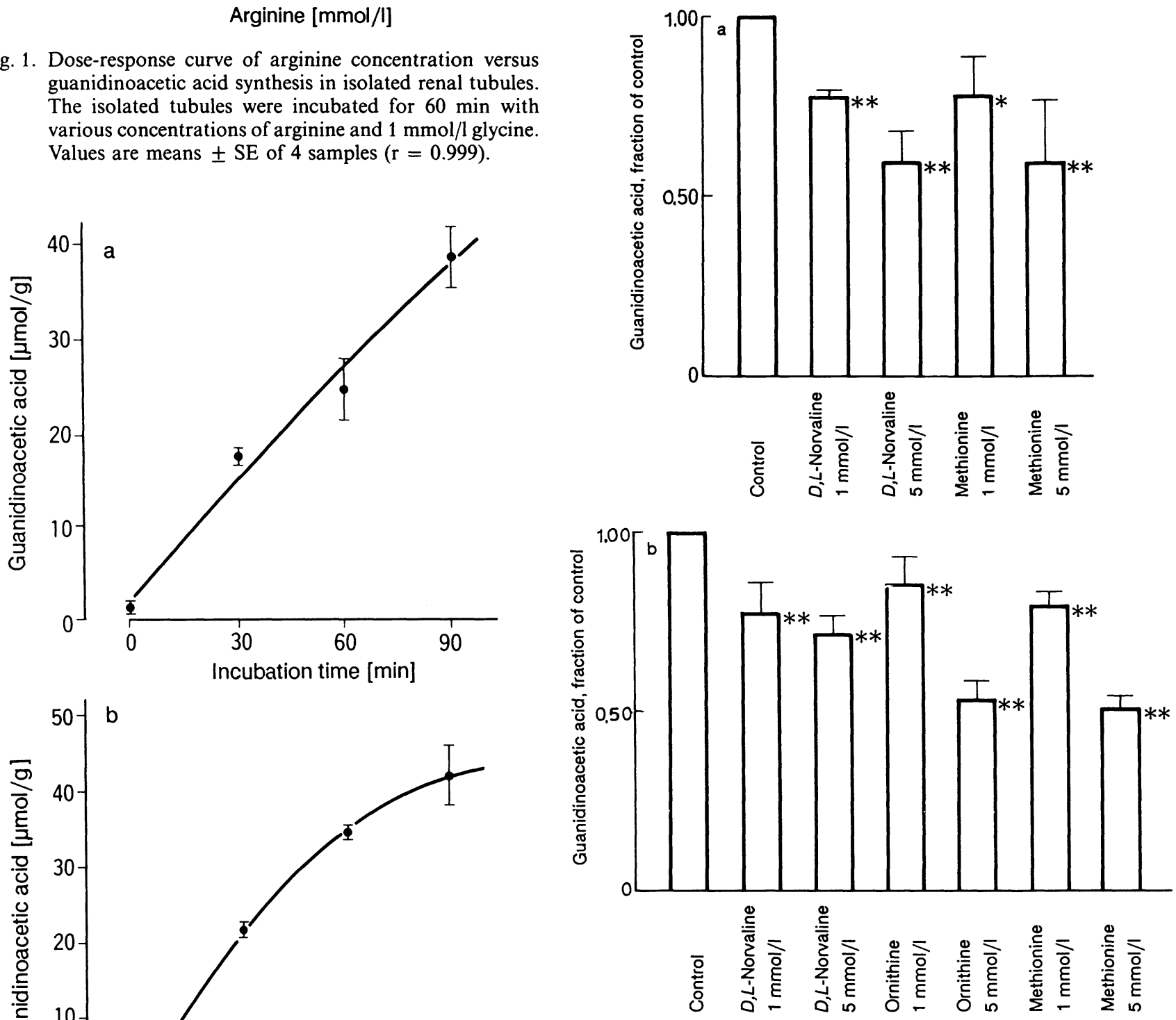

Fig. 3. Regulation of guanidinoacetic acid synthesis by various substrates in isolated rat tubules.

(a) The isolated tubules were incubated with $1 \mathrm{mmol} / \mathrm{l}$ arginine and $1 \mathrm{mmol} / \mathrm{l}$ glycine with or without 1 or $5 \mathrm{mmol} / \mathrm{l} D, L$-norvaline and methionine for $60 \mathrm{~min}$.

(b) 1 or $5 \mathrm{mmol} / \mathrm{l} D, L$-norvaline, ornithine and methionine were added to the isolated renal tubules incubated with $1 \mathrm{mmol} / \mathrm{l}$ canavanine and $1 \mathrm{mmol} / \mathrm{l}$ glycine for 60 min.

Values are means $\pm \mathrm{SE}$ of 5 samples.

$* \mathrm{P}<0.05, \quad * * \mathrm{P}<0.01$ 
guanidinoacetic acid synthesis from arginine and glycine. The isolated renal tubules were incubated with $1 \mathrm{mmol} / \mathrm{l}$ arginine or $1 \mathrm{mmol} / \mathrm{l}$ canavanine and $1 \mathrm{mmol} / \mathrm{l}$ glycine with or without the addition of 1 or $5 \mathrm{mmol} / \mathrm{l} D, L$-norvaline, ornithine or methionine. As shown in figure $3 \mathrm{a}, 1$ or $5 \mathrm{mmol} / \mathrm{l} D, L$-norvaline and methionine significantly suppressed guanidinoacetic acid synthesis from arginine and glycine as substrates. Figure $3 \mathrm{~b}$ shows that 1 or $5 \mathrm{mmol} / 1 D, L$-norvaline, ornithine and methionine significantly inhibited guanidinoacetic acid synthesis from canavanine and glycine.

\section{Effect of various concentrations of creatine} on guanidinoacetic acid synthesis in the isolated renal tubules

We added various concentrations of creatine to the isolated renal tubules incubated with arginine or canavanine and glycine. The addition of 100 and 300 $\mathrm{mg} / \mathrm{l}$ of creatine suppressed guanidinoacetic acid synthesis from arginine and glycine as substrates (fig. 4).

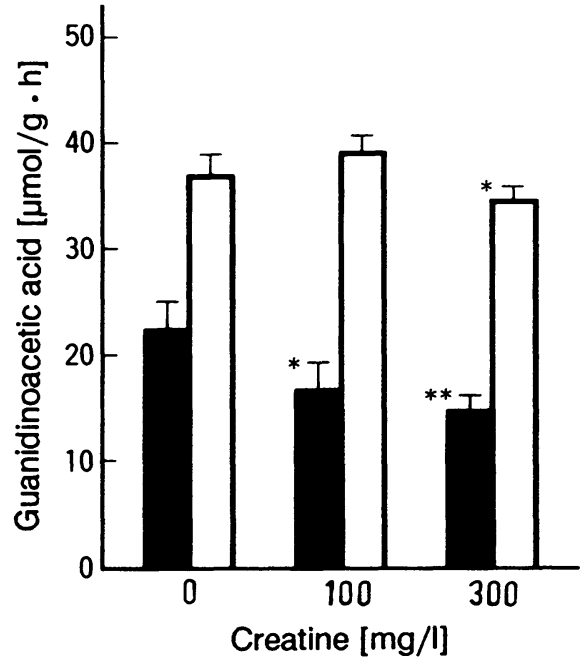

Fig. 4. Effect of creatine on guanidinoacetic acid synthesis in isolated rat tubules. Various concentrations of creatine were added to isolated tubules incubated with $1 \mathrm{mmol} / \mathrm{l}$ arginine $+1 \mathrm{mmol} / \mathrm{l}$ glycine ( $(\mathbf{a})$ or $1 \mathrm{mmol} / \mathrm{l}$ canavanine $+1 \mathrm{mmol} / \mathrm{l}$ glycine ( $\square$ ) for $60 \mathrm{~min}$. Values are means \pm SE of 5 samples.

* $\mathrm{P}<0.05,{ }^{* *} \mathrm{P}<0.01$.

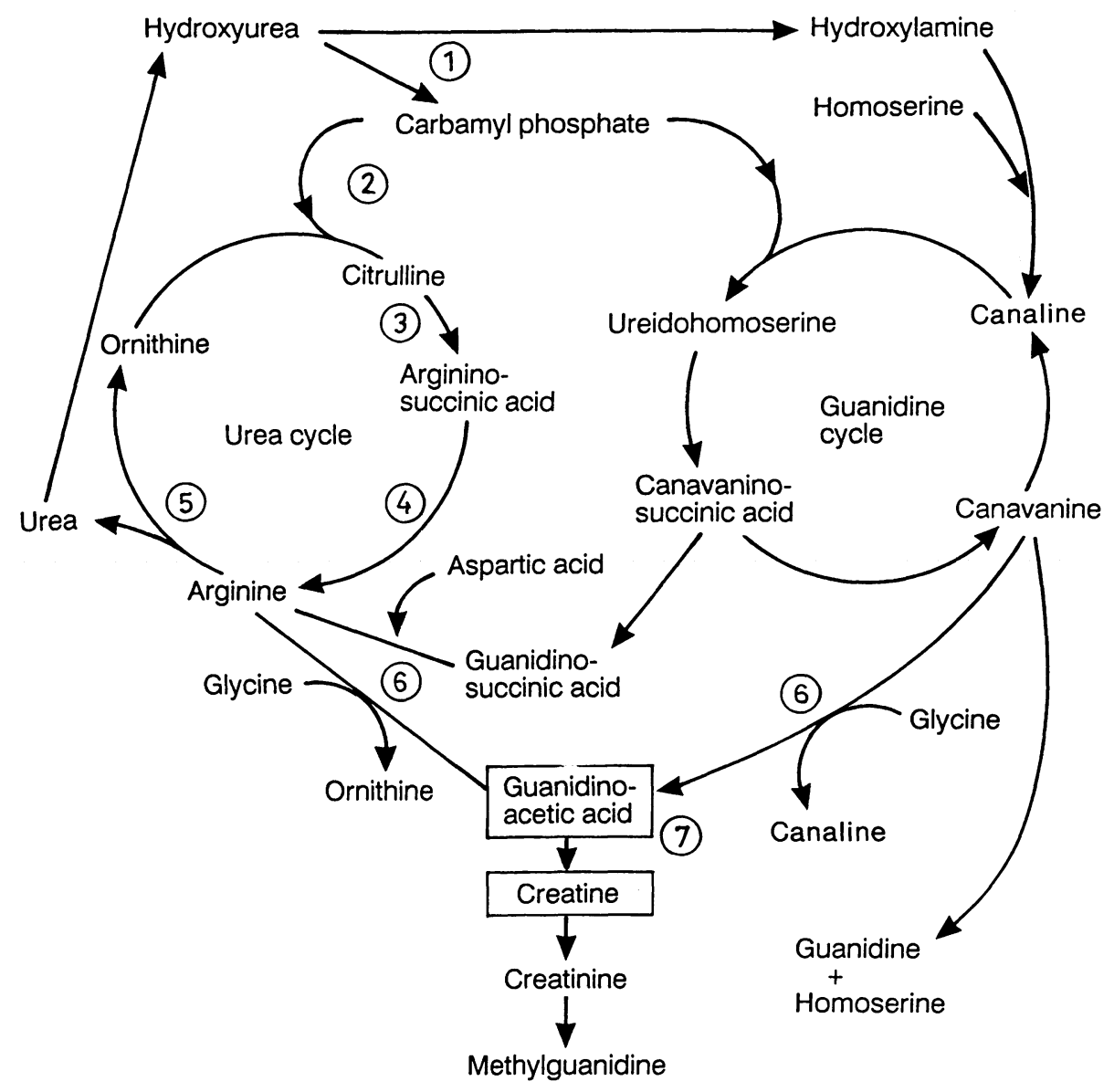

Fig. 5. Metabolic reactions of the guanidine and urea cycles.

Enzymes
(1) Carbamylphosphate synthetase
(2) Ornithine transcarbamylase
(3) Argininosuccinic acid synthetase
(4) Argininosuccinic acid lyase
(5) Arginase
(6) Glycine-amidinotransferase (transamidinase)
(7) Guanidinoacetate methyltransferase 
When canavanine and glycine were used as substrates, 100 and $300 \mathrm{mg} / \mathrm{l}$ of creatine also significantly suppressed guanidinoacetic acid synthesis. These results suggest the negative feedback control of guanidinoacetic acid synthesis by creatine in vitro.

Possible metabolic pathway of guanidinoacetic acid synthesis in the isolated renal tubules

Among the urea cycle enzymes shown in figure 5, ornithine transcarbamylase ${ }^{1}$ ), argininosuccinic acid synthetase and argininosuccinic acid lyase ${ }^{1}$ ) have been reported to exist in the renal medulla at low levels $(26,27)$. On the other hand, there has been no evidence as to whether the guanidine cycle exists in the kidney, while it is considered to exist in the liver (28). We tried to determine the presence of enzymes of the urea and guanidine cycles in isolated renal tubules by determining guanidinoacetic acid synthesis. We incubated the isolated renal tubules with $10 \mathrm{mmol} / \mathrm{l}$ glycine and $1 \mathrm{mmol} / \mathrm{l}$ of either arginine, canavanine, canaline, hydroxyurea, citrulline or argininosuccinic acid.

As shown in figure 6, guanidinoacetic acid was clearly synthesized from arginine and canavanine, but there was no synthesis from hydroxyurea, citrulline, agrininosuccinic acid or canaline.

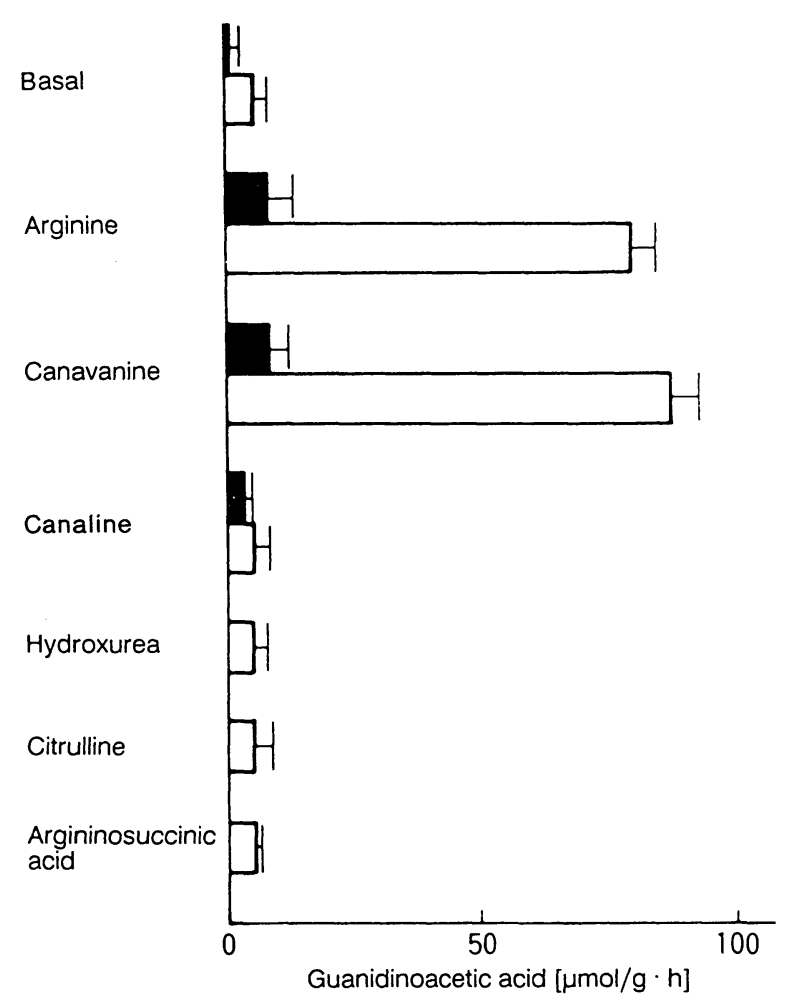

Fig. 6. Guanidinoacetic acid synthesis in isolated rat tubules from various substrates. The isolated tubules were incubated without ( $(\mathbf{\square})$ and with $10 \mathrm{mmol} / 1$ glycine ( $\square$ ) and $1 \mathrm{mmol} / \mathrm{l}$ of various substrates indicated on the ordinate for $60 \mathrm{~min}$. Values are means $\pm \mathrm{SE}$ of 4 samples.

\section{Discussion}

Creatine is synthesized according to the following two steps.

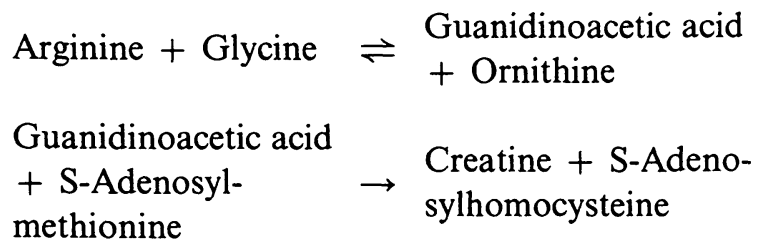

Transamidinase ${ }^{1}$ ) and guanidinoacetate-methyltransferase $\left.{ }^{1}\right)$ catalyse reactions $[A]$ and $[B]$, respectively $(1,2)$. Arginine in reaction $[\mathrm{A}]$ has been reported to be synthesized from citrulline mainly in the kidney $(29,30)$. Reaction [A] also occurs mainly in the kidney (15).

Guanidinoacetic acid synthesis in the kidney has been investigated with rat kidney homogenates $(10-15)$ and slices $(1,16)$. Although the numerous previous studies on guanidinoacetic acid synthesis used kidney homogenates $(10-15)$, the present studies were performed on isolated renal tubules consisting mainly of proximal convoluted tubule, where transamidinase was observed by an immunofluorescence technique (31). Furthermore, the specificity and sensitivity of our HPLC and fluorometric methods (21) permit the determination of lower levels of guanidinoacetic acid synthesis from low substrate concentrations, compared with previous studies $(1,10-15)$. Thus, it is likely that the guanidinoacetic acid synthesis measured in the present study reflects a more physiological metabolism.

Transamidinase activity has been shown to be regulated by dietary $(16,17)$ or hormonal factors $(17)$, in vivo. However, the regulation of guanidinoacetic acid synthesis by various substrates has never been fully investigated in renal tubules. In this study, we demonstrated the effect of various substrates on the regulation of guanidinoacetic acid synthesis in isolated renal tubules. $D, L$-Norvaline, a multiple enzyme inhibitor of the urea cycle (32), inhibited guanidinoacetic acid synthesis in isolated renal tubules, This finding is consistent with the data in isolated hepatocytes (25) and human pancreas homogenates incubated with canavanine and glycine as substrates (24). When arginine and glycine were used as substrates, however, our results were not consistent with the data in isolated rat hepatocytes, in which $D, L$-norvaline did not inhibit guanidinoacetic acid synthesis (25). This study may provide the first evidence of the suppression of transamidinase activity by $D, L$-norvaline. Ornithine, a member of urea cycle that promotes urea synthesis (33), has been reported to suppress guanidinoacetic 
acid synthesis from arginine and glycine in rat kidney homogenates (10). This may be a simple negative feedback control of guanidinoacetic acid synthesis. We found that ornithine inhibited transamidinase activity by using canavanine and glycine as substrates. Furthermore, we found that methionine also suppressed guanidinoacetic acid synthesis in isolated renal tubules. It is highly likely that guanidinoacetic acid synthesized from arginine or canavanine and glycine is methylated to creatine. However, as reported previously, we failed to detect creatine synthesis in microdissected proximal convoluted tubules, isolated glomeruli or kidney homogenates with guanidinoacetic acid or arginine and glycine plus methionine (34). At present, we postulate that methionine may have an inhibitory effect on transamidinase activity in the kidney.

It has been reported that creatine feeding suppresses transamidinase activity in rat kidney homogenates (16). Transamidinase is considered to be a rate limiting enzyme for creatine synthesis. In contrast, Fitch et al. (20) have reported that the addition of creatine did not inhibit transamidinase activity in rat kidney homogenates. In our study, the addition of $300 \mathrm{mg} / 1$ creatine, which is about $1 / 110$ of the concentration used in Fitch's study, suppressed guanidinoacetic acid synthesis when either arginine or canavanine were present as the amidine donor. The mechanism of the decrease of transamidinase activity by creatine feeding is of considerable interest. $M c$ Guire et al. (35) reported that the relative rate of synthesis and the mRNA levels of transamidinase were reduced in creatine-fed rats. Thus, creatine was thought to inhibit transamidinase activity by affecting its rate of synthesis at a pretranslational stage. It is unlikely that creatine could be a signal for mRNA functional activities in kidney homogenates (35), in which the cell structures are destroyed. In contrast, in our isolated renal tubules, where cell structures were preserved, the addition of creatine may reduce transamidinase activity at a pretranslational stage.

Among the urea cycle enzymes in the kidney, ornithine transcarbamylase, argininosuccinic acid synthetase

\section{References}

1. Borsook, H. \& Dubnoff, J. W. (1941) The formation of glycocyamine in animal tissues. J. Biol. Chem. 138, 389403.

2. Gerber, G. B., Gerber, G., Koszalka, T. R. \& Miller, L. L. (1962) The rate of creatine synthesis in the isolated, perfused rat liver. J. Biol. Chem. 237, 2246-2250.

3. Sasaki, M., Takahara, K. \& Natelson, S. (1973) Urinary guanidinoacetate/guanidinosuccinate ratio: an indicator of kidney dysfunction. Clin. Chem. 19, 315-231. and argininosuccinic acid lyase are present in the medulla in very small amounts $(26,27)$, and the distribution of carbamylphosphate synthetase is reported to coincide with ornithine transcarbamylase activity (27). The fact that guanidinoacetic acid could not be produced from hydroxyurea may confirm the data that only very small amounts of carbamylphosphate synthetase are present (27). Since our isolated tubules consisted mainly of cortical proximal tubules and since argininosuccinic acid synthetase is located mainly in the medulla, as stated above (26), it seems reasonable that citrulline plus glycine did not produce guanidinoacetic acid. In contrast, Funahashi et al. (29) and Featherston et al. (30) have reported that the kidney synthesizes a large portion of endogenous arginine from citrulline in vivo. In these studies, citrulline was converted by argininosuccinic acid synthetase and argininosuccinic acid lyase in the renal medulla. Recently, Saheki et al. (36) used the immunofluorescence technique to show that argininosuccinic acid synthetase is localized in the cell structures of proximal convoluted tubules. Even if argininosuccinic acid synthetase is located in the proximal convoluted tubules, guanidinoacetic acid synthesis from citrulline might not be recognized because argininosuccinic acid lyase is localized mainly in the medulla (26). In our studies, guanidinoacetic acid synthesis from argininosuccinic acid and glycine was not observed in renal tubules. We confirmed existing information on the presence of urea cycle enzymes in the kidney by determining guanidinoacetic acid synthesis in isolated rat tubules. The guanidine cycle has been proposed to exist in the liver and to interrelate with the urea cycle (28). Canaline, which is part of the guanidine cycle (28), did not produce guanidinoacetic acid in isolated rat tubules in our studies. From these results, it is highly likely that the entire guanidine cycle or certain enzymes in this cycle are depleted in the rat kidney.

\section{Acknowledgement}

This study was supported in part by grants from the Japanese Ministry of Education, Science and Culture and the Japanese Ministry of Health and Welfare.
4. Alsever, R. N., Georg, R. H. \& Sussman, K. E. (1970) Stimulation of insulin secretion by guanidinoacetic acid and other guanidine derivatives. Endocrinology 86, 332336.

5. Tsubakihara, Y., Iida, N., Yuasa, S., Kawashima, T., Nakanishi, I., Tomobuchi, M., Yokogawa, T., Ando, A., Orita, T., Kikuchi, T. \& Okamoto, H. (1985) Guanidinoacetic acid (GAA) deficiency and supplementation in rats with chronic renal failure (CRF). In: Guanidines (Mori, A., Cohen, B. 
D. \& Lowenthal, A., eds.) pp. 373-379, Plenum, New York.

6. Nakayama, S., Kiyatake, I., Shirokane, Y. \& Koide, H. (1990) Effect of antibiotic administration of urinary guanidinoacetic acid excretion in renal disease. In: Guanidines II (Mori, A., Cohen, B. D. \& Koide, H., eds.) pp. 313322, Plenum, New York.

7. Nakayama, S., Junen, M., Kiyatake, I. \& Koide, H. (1990) Urinary guanidinoacetic acid (U-GAA) as an indicator of gentamicin nephrotoxicity in rats. In: Guanidines II (Mori, A., Cohen, B. D. \& Koide, H., eds.) pp. 303-311, Plenum, New York.

8. Takano, Y., Aoike, I., Gejo, F. \& Arakawa, M. (1989) Urinary excretion rate of guanidinoacetic acid as a new marker in hypertensive renal damage. Nephron 52, 273277.

9. Ishizaki, M., Kitamura, H., Takahashi, H., Asano, H., Miura, K. \& Okazaki, H. (1985) Evaluation of the efficacy of anti-rejection therapy using the quantitative analysis of guanidinoacetic acid (GAA) urinary excretion as a guide. In: Guanidines (Mori, A., Cohen, B. D. \& Lowenthal, A., eds.) pp. $353-363$, Plenum, New York.

10. Ratner, S. \& Rochovansky, O. (1956) Biosynthesis of guanidinoacetic acid. I. Purification and properties of transamidinase. Arch. Biochem. Biophys. 63, 277-295.

11. Nakatsu, S. (1956) Enzymatic transamidination from canavanine to glycine by hog kidney extracts. J. Biochem. 43, 675-681.

12. Van Pilsum, J. F., Berman, D. A. \& Wolin, E. A. (1957) Assay and some properties of kidney transamidinase. Proc. Soc. Exp. Biol. Med. 43, 675-681.

13. Conconi, F. \& Grazi, E. (1965) Transamidinase of hog kidney. I. Purification and properties. J. Biol. Chem. 240, $2461-2464$.

14. Van Pilsum, J. F., Taylor, D., Zakis, B. \& McCormick, P. (1970) Simplified assay for transamidinase activities of rat kidney homogenates. Anal. Biochem. 35, 277-286.

15. Van Pilsum, J. F., Stephens, G. C. \& Taylor, D. (1972) Distribution of creatine, guanidinoacetate and the enzymes for their biosynthesis in the animal kingdom. Biochem. J. $126,325-345$.

16. Walker, J. B. (1979) Creatine: biosynthesis, regulation and function. Adv. Enzymol. 52, 177-242.

17. Van Pilsum, J. F. \& Canfield, T. M. (1962) Creatine: biosynthesis, regulation and function. J. Biol. Chem. 237, 2574-2577.

18. Ungar, F. \& Van Pilsum, J. F. (1966) Hormonal regulation of rat kidney transamidinase; effect of growth hormone in the hypophysectomized rat. Endocrinology 78, 1238-1247.

19. Fitch, C. D., Hus, C. \& Dinning, J. S. (1960) Some factors affecting kidney transamidinase activity in rats. J. Biol. Chem. 235, 2362-2364.

20. Obara, T., Yamada, H., Nakada, J. \& Endou, H. (1985) Atriatic natriuretic peptides stimulate gluconeogenesis. Biochem. Biophys. Res. Commun. 129, 833-839.
21. Yamamoto, Y., Manji, T., Saito, A., Maeda, K. \& Ohta, K. (1979) Ion-exchange chromatographic separation and fluorometric detection of guanidino compounds in physiologic fluids. J. Chromatogr. 162, 327-340.

22. Lowry, O. H., Rosenbrought, N. J., Farr, A. L. \& Randall, R. J. (1951) Protein measurement with the folin phenol reagent. J. Biol. Chem. 193, 265-275.

23. Colquhoun, D. (1971) Lectures on Biostatistics. Oxford University Press.

24. Walker, J. B. (1963) Formamidine group transfer in extracts of human pancreas, liver, and kidney. Biochem. Biophys. Acta 73, 241-247.

25. Aoyagi, K., Ohba, S., Miyazaki, M., Nagase, S., Iida, S., Narita, M. \& Tojo, S. (1985) Biosynthesis of guanidinoacetic acid in isolated rat hepatocytes. In: Guanidines (Mori, A., Cohen, B. D. \& Lowenthal, A., eds.) pp. 83-92, Plenum, New York.

26. Szepesi, B., Avery, H. \& Freeland, R. A. (1970) Role of kidney in gluconeogenesis and amino acid catabolism. Am. J. Physiol. 219, 1627-1631.

27. Raijman, L. (1974) Citrulline synthesis in rat tissues and liver content of carbamyl phosphate and ornithine. Biochem. J. 138, 225-232.

28. Natelson, S. \& Sherwin, J. E. (1979) Proposed mechanism for urea nitrogen re-utilization: relationship between urea and proposed guanidine cycles. Clin. Chem. 25, 13431344.

29. Funahashi, M., Kato, H., Shiosaka, S. \& Nakagawa, H. (1981) Formation of arginine and guanidinoacetic acid in vivo. Their relations with the liver and their regulation. J. Biochem. Tokyo 89, 1347-1356.

30. Featherston, W. R., Rogers, Q. R. \& Freedland, R. A. (1973) Relative importance of kidney and liver in synthesis of arginine by rat. Am. J. Physiol. 224, 127-129.

31. McGuire, D. M., Gross, M. D., Elde, R. P. \& Van Pilsum, J. F. (1986) Localization of L-arginine-glycine amidinotransferase protein in rat tissues by immunofluorescence microscopy. J. Histochem. Cytochem. 34, 429-435.

32. Saheki, T., Sato, Y., Takada, S. \& Katsunuma, T. (1979) Regulation of urea synthesis in rat liver. Inhibition of urea synthesis by L-norvaline. J. Biochem. Tokyo 86, 745-750.

33. Saheki, T. \& Katsunuma, N. (1975) Analysis of regulatory factors for urea synthesis by isolated perfused rat liver. J. Biochem. 77, 659-669.

34. Takeda, M., Jung, K. Y., Endou, H. \& Koide, H. (1992) Intranephron distribution of creatine content in rats. Renal Physiol. Biochem., in press.

35. McGuire, D. M., Gross, M. D., Van Pilsum, J. F. \& Towel, H. C. (1984) Repression of rat kidney L-arginine: glycine amidinotransferase synthesis by creatine at a pretranslational level. J. Biol. Chem. 259, 12034-12038.

36. Saheki, T., Sase, M., Nakano, K. \& Yagi, Y. (1985) Arginine metabolism in citrullinemic patients. In: Guanidines (Mori, A., Cohen, B. D. \& Lowenthal, A., eds.) pp. 149-158, Plenum, New York.

Hikaru Koide, M.D.
Division of Nephrology
Department of Medicine
Juntendo University
School of Medicine
2-1-1 Hongo, Bunkyo-ku
Tokyo 113
Japan

Hikaru Koide, M. D.

Division of Nephrology

Juntendo University

School of Medicine

Tokyo 113

Japan 
